\title{
Phase-contrast imaging using radiation sources based on laser-plasma wakefield accelerators: state of the art and future development
}

\author{
D. Reboredo*a, S. Cipiccia a , P. A. Grant ${ }^{\mathrm{a}}$, G. H. Welsh ${ }^{\mathrm{a}}$, D. W. Grant ${ }^{\mathrm{a}}$, G. McKendrick ${ }^{\mathrm{a}}$, A. Subiel ${ }^{\mathrm{b}}$, \\ D. Maneuskic , S. M. Wiggins ${ }^{a}$, D. A. Jaroszynski ${ }^{a, \dagger}$ \\ ${ }^{a}$ University of Strathclyde, Department of Physics, Scottish Universities Physics Alliance Glasgow, \\ G4 0NG (UK); ${ }^{b}$ National Physical Laboratory, Acoustics and Ionising Radiation, Teddington, TW11 \\ OLW (UK); ' University of Glasgow, School of Physics and Astronomy, Scottish Universities \\ Physics Alliance, Glasgow, G12 8QQ, (UK)
}

\begin{abstract}
Both the laser-plasma wakefield accelerator (LWFA) and X-ray phase-contrast imaging (XPCi) are promising technologies that are attracting the attention of the scientific community. Conventional X-ray absorption imaging cannot be used as a means of imaging biological material because of low contrast. XPCi overcomes this limitation by exploiting the variation of the refraction index of materials. The contrast obtained is higher than for conventional absorption imaging and requires a lower dose. The LWFA is a new concept of acceleration where electrons are accelerated to very high energy $(\sim 150$ $\mathrm{MeV}$ ) in very short distances (mm scale) by surfing plasma waves excited by the passage of an ultra-intense laser pulse $\left(\sim 10^{18} \mathrm{Wcm}^{-2}\right)$ through plasma. Electrons in the LWFA can undergo transverse oscillation and emit synchrotron-like (betatron) radiation in a narrow cone around the propagation axis. The properties of the betatron radiation produced by LWFA, such as source size and spectrum, make it an excellent candidate for XPCi. In this work we present the characterization of betatron radiation produced by the LWFA in the ALPHA-X laboratory (University of Strathclyde). We show how phase contrast images can be obtained using the betatron radiation in a free-space propagation configuration and we discuss the potential and limitation of the LWFA driven XPCi.
\end{abstract}

Keywords: X-ray imaging, phase-contrast, betatron radiation, lasers, plasmas, electron beams.

\section{INTRODUCTION}

Currently, most clinical medical imaging is based on conventional X-ray absorption ${ }^{1}$. The main drawbacks of these techniques are the high dose delivered to tissue and the low contrast due to low absorption. XPCi overcomes these deficiencies enhancing the contrast of weakly absorbing tissue.

XPCi relies on the phase shift term $(\delta)$ of the refractive index $n=1-\delta+i \beta$, whereas conventional imaging is based on the absorption term $(\beta)$.

For most biological tissue (in the $40-150 \mathrm{keV}$ energy range used for clinical medical imaging), $\delta$ is around 1000 times larger than $\beta$. This enables the detection of detail that is invisible to conventional absorption techniques. Because of this, XPCi has been widely studied for use in clinical medical imaging applications ${ }^{2}$.

However, the progress in this field has been impeded because the spatially coherent sources required to perform XPCi experiments demand synchrotron radiation sources, which have limited access due to the high demand for beam time. Although great progress has been made using incoherent sources such as X-ray tubes ${ }^{3}$, this problem can be overcome using the laser-plasma wakefield accelerator ${ }^{4-6}$ (LWFA) systems, which have experienced a rapid development in recent years and represent a potential alternative to conventional synchrotron sources.

The unique properties of both electron and X-ray beam sources from a LWFA can be used in a wide range of applications such as XPCi, radiobiology and isotope generation etc. X-ray sources from the LWFA represent a good candidate for XPCi experiments because they have the required degree of spatial coherence because of their micrometer-scale source size ${ }^{7}$.

*david.reboredo-gil@strath.ac.uk, †d.a.jaroszynski@strath.ac.uk 


\section{LASER WAKEFIELD ACCELERATION: BETATRON RADIATION}

Plasma is a suitable medium to sustain high accelerating gradients since it is not limited by electrical breakdown such as in conventional accelerators. In plasma acceleration particles gain energy from longitudinal plasma waves and several schemes can be used to produce plasma waves, although the most successful one is the LWFA proposed by Tajima and Dawson ${ }^{8}$.

The invention of chirped-pulse amplification ${ }^{9}$ enabled table-top femtosecond lasers to reach intensities as high as $10^{18} \mathrm{~W}$ $\mathrm{cm}^{-2}$, which has resulted in stable high-quality monoenergetic electron beams of hundreds of MeV with small energy spread $^{10}$, low emittance ${ }^{11}$ and high charge ${ }^{6}$. These characteristics from LWFAs are routinely produced in laboratories worldwide. These properties also make LWFAs an attractive candidate for driving a free-electron laser ${ }^{12}$.

Focusing a high power laser into underdense plasma, $\omega_{o} \gg \omega_{p}$, where $\omega_{o}$ is the laser frequency, $\omega_{p}=\sqrt{4 \pi n_{p} e^{2} / m_{e}}$ the plasma frequency, $e$ and $m_{e}$ are the charge and mass of electrons and $n_{p}$ the electron density The passage of the laser pulse drives electron density oscillations and a plasma wave is formed. If the laser pulse is matched to the plasma wavelength a blowout region or ion bubble ${ }^{13}$ is formed behind the laser, leading to large linear accelerating fields. These fields can trap electrons and result in $\mathrm{GeVcm}^{-1}$ acceleration gradients, three orders of magnitude larger than conventional RF cavities used in conventional accelerators.

This large accelerating gradient makes the LWFA an ideal alternative to conventional acceleration because it allows miniaturization of the accelerators by a factor greater than 1000. These compact "table-top" accelerators are particularly attractive for applications in different fields such as radiobiology, probing dense matter, radioisotope production, nuclear physics and medical imaging.

Moreover, a synchrotron-like source is embedded in the LWFA. While electrons are accelerated in the forward direction, their transverse momentum leads to transverse (betatron) oscillations, similar to in a wiggler, because of the radial electrostatic fields of the plasma bubble, which leads to the emission of bright synchrotron-like X-rays (betatron X-rays ${ }^{14}$ ). The betatron frequency is given by:

$$
\omega_{\beta}=\frac{\omega_{p}}{\sqrt{2 \gamma}},
$$

where $\gamma$ is the Lorenz factor of the electron.

The characteristics of the betatron emission depend on the amplitude of the betatron oscillations $r_{\beta}$. For small betatron oscillations radiation is emitted at the fundamental frequency:

$$
\omega=\sqrt{2} \gamma^{3 / 2} \omega_{p}
$$

and when the amplitude of the oscillations is large the spectrum of the radiation emitted is broadband and extends up to a critical energy after which it drops exponentially. The critical energy ${ }^{15}$ is given by:

$$
E_{c}=\frac{3 \hbar \omega_{p}^{2} \gamma^{2} r_{\beta}}{2 c}
$$

The radiation is emitted in the forward direction within a half-angle:

$$
\vartheta=\frac{a_{\beta}}{\gamma}
$$

where $a_{\beta}$ is the normalized transverse momentum. The number of photons per electron emitted every betatron oscillation is proportional to $\gamma^{2}$ and is determined by the Lienard-Wiechert ${ }^{15}$ potentials:

$$
N_{\text {phot }}=\frac{2 \pi \alpha_{f} a_{\beta}}{9} \text {. }
$$

\section{CHARACTERIZATION OF THE STRATHCLYDE X-RAY SOURCE}

Prior to exploring imaging applications the betatron X-ray source produced in the ALPHA-X laser wakefield accelerator beam line ${ }^{16}$ at the University of Strathclyde has been characterized. For this purpose laser pulses of $0.9 \mathrm{~J}, 35 \mathrm{fs}$, and a 
spectrum centered at $800 \mathrm{~nm}$, from a Ti:sapphire laser are focused using a F/18 spherical mirror to a spot diameter of $40 \mu \mathrm{m}\left(1 / \mathrm{e}^{2}\right)$ at the entrance of a $2.7 \mathrm{~mm}$ diameter pulsed supersonic helium gas jet producing a $10 \mu \mathrm{m}$ wide relativistic plasma channel. Those laser parameters correspond to a peak intensity of $I=2 \times 10^{18} \mathrm{~W} / \mathrm{cm}^{2}$ on target.

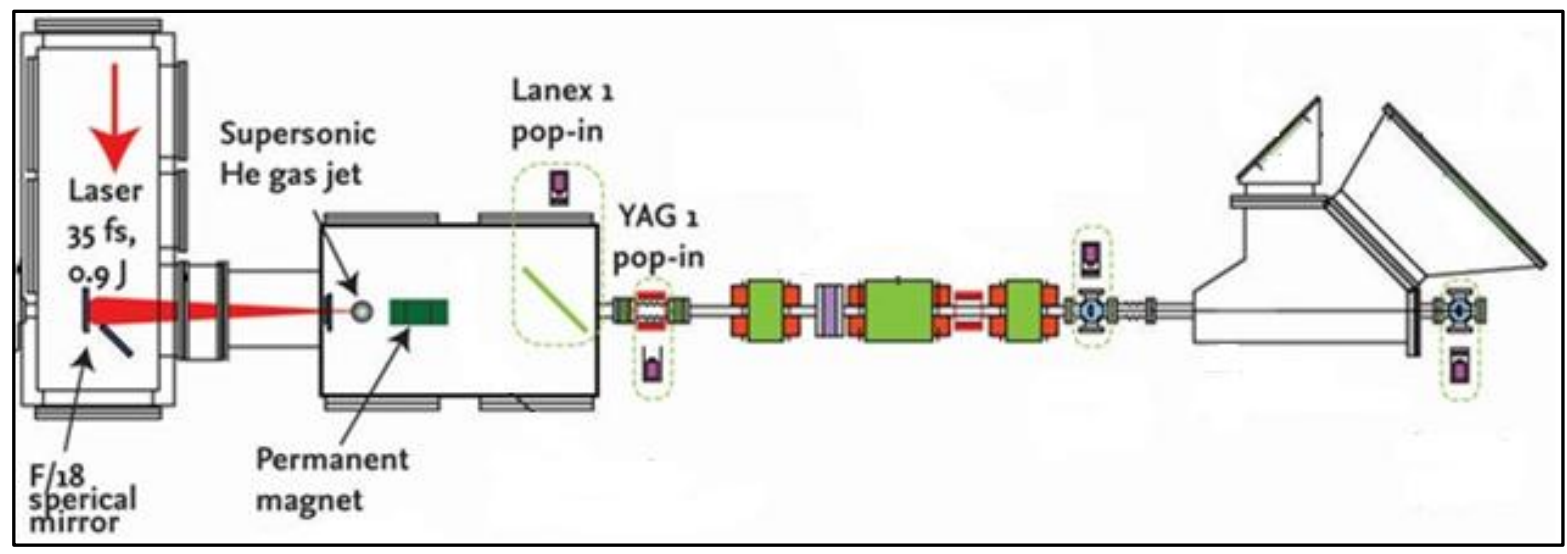

Figure 1. Layout of the ALPHA-X beam line. A $0.9 \mathrm{~J}$ and 35 fs high power laser is focused onto a gas jet to produce a monoenergetic stable electron beam which is imaged on a LANEX screen.

A $0.7 \mathrm{~T}$ bending magnet is placed inside the interaction chamber just after the gas-jet to deflect the accelerated electrons away from the laser axis, allowing the betatron X-rays to propagate along the beamline. The introduction of the magnet minimizes the on-axis bremsstrahlung component, which reduces the background noise of the X-ray detector. This is placed downstream to characterize the betatron radiation. The X-ray beam leaves the interaction chamber and propagates through a column of air to two different X-ray detectors: an imaging plate and a semiconductor detector.

The deflected electrons are detected using a scintillating LANEX phosphor screen and their energy spectrum is measured. Stable quasi-monoenergetic electron beams of $\sim 150 \mathrm{MeV}$ are measured. Charge between 5-10 pC/shot is measured using imaging plates. The typical bunch length of the electron beams from a LWFA are in the range 1-10 fs ${ }^{17}$. The repetition rate of the accelerator is set to $0.33 \mathrm{~Hz}$, limited by gas loading on the vacuum pumps.

The measurement of the critical energy $\left(\mathrm{E}_{\mathrm{c}}\right)$ of the betatron radiation is performed using sets of different metal filters $(\mathrm{Cu}$, $\mathrm{Al}$, Ti and Ag). The shadows cast by the metal filters are recorded with imaging plates Fuji BAS-SR. The YAG 1 pop-in is removed and both metal filters and imaging plate are inserted instead (see Figure 1). From the measured absorption of the X-ray beam, the critical energy is estimated. All the measurements are performed in a single shot. The average critical energy estimated for the run is $\sim 15 \mathrm{keV}$.
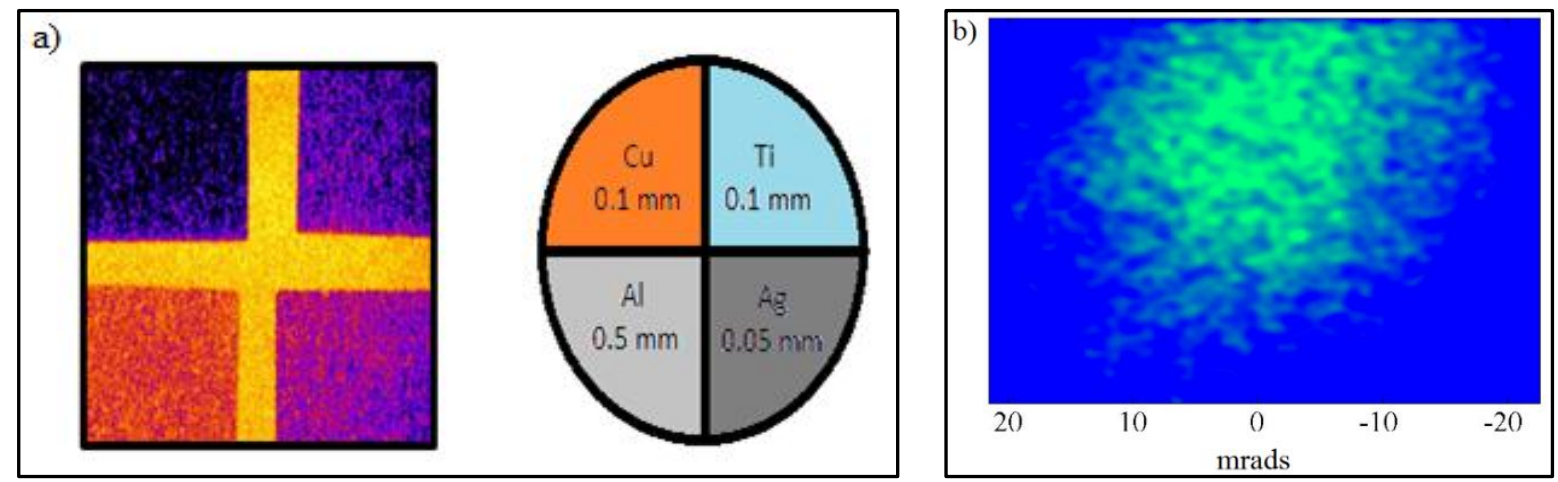

Figure 2. (a) Shadow left by the metal filters in a imaging plate on a typical shot and distribution of the thickness and material used to measure the critical energy. (b) Typical shot showing the spatial distribution of the X-ray source.

A decrease in the critical energy is observed by increasing the plasma density. When the plasma density is increased the energy of the electron beam decreases and, therefore, the critical energy of the betatron X-rays decreases which is a simple technique for tuning the X-ray source. Plasma densities, obtained by characterizing the gas jet using a Mach-Zehnder 
interferometer, were in the range from $1.7 \cdot 10^{19}$ electrons $/ \mathrm{cm}^{3}$ to $2.4 \cdot 10^{19}$ electrons $/ \mathrm{cm}^{3}$ depending on the gas backing pressure used.

The total number of photons has been measured using the Timepix silicon detector. Timepix is a high spatial resolution and high contrast semiconductor detector working in single photon counting mode. It is formed by a matrix of $256 \times 256$ pixels of $55 \times 55 \mu \mathrm{m}$ size. When ionizing radiation is incident on the detector, a signal proportional to the charge generated by the passage of the radiation is produced thus, enabling spectroscopic measurements. More details on this detector can be found elsewhere ${ }^{18}$.

Table 1 . Summary of the critical energy measurements at different plasma densities.

\begin{tabular}{c|c|c|c|c}
$\boldsymbol{n}_{p}\left(\mathbf{1 0}^{\mathbf{1 9}} \mathbf{~ c m}^{-\mathbf{3}}\right)$ & $1.72 \pm 0.17$ & $2.04 \pm 0.20$ & $2.24 \pm 0.22$ & $2.40 \pm 0.24$ \\
\hline $\boldsymbol{E}_{\boldsymbol{c}}(\mathbf{k e V})$ & $17.7 \pm 2.5$ & $15.7 \pm 1.2$ & $15.8 \pm 1.2$ & $14.9 \pm 1.2$
\end{tabular}

During the experiment the flux of photons on the detector is kept below a single photon per pixel by reducing the flux using the air column and additional metal filters. As in the previous experiment, the electron beam is deflected with a bending magnet and the X-rays left to propagate through $4.5 \mathrm{~m}$ of air before reaching the detector. A total flux of $10^{7}$ photons/shot is measured.

The spatial distribution and the divergence of the X-ray beam is measured by imaging the full X-ray beam just after the interaction chamber using imaging plates. This measurement is carried out at the same place as the critical energy measurement. From this measurement, an average full angle divergence of $\sim 40 \mathrm{mrad}$ is obtained in the horizontal axis and $\sim 35 \mathrm{mrad}$ in the vertical axis.

\section{PHASE-CONTRAST IMAGING USING BETATRON RADIATION ON THE ALPHA-X BEAMLINE}

To demonstrate the potential of a LWFA driven X-ray source for XPCi, a proof of principle experiment has been carried out using the betatron beam produced on the ALPHA-X beamline in a free space propagation configuration ${ }^{19}$. Similar experiments have been performed before ${ }^{20}$ although using more powerful lasers and different distances source-sample and sample-detector. The only requirements for this experimental configuration are a high spatially coherent source and an Xray detector. Monochromatic X-rays are not required.

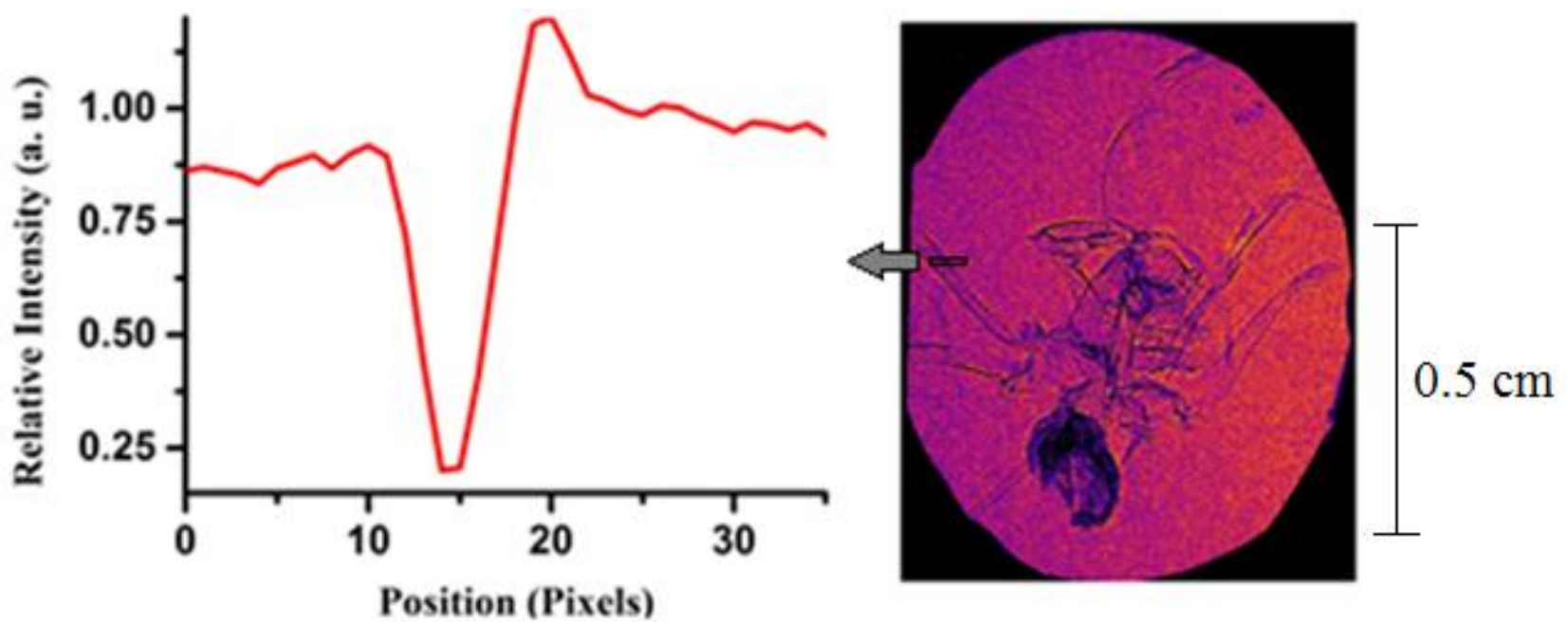

Figure 3. 1.h.s: image obtained after 300 shots. r.h.s: relative intensity from the edge of a leg. Contrast is estimated to be 0.63 . The image is obtained after 300 shots at a repetition rate of $0.33 \mathrm{~Hz}$.

For the experiment a biological sample (dead domestic spider) is placed just after the interaction chamber and imaged with an imaging plate. The sample is placed at a source-object distance $\left(R_{1}\right)$ of $0.82 \mathrm{~m}$. The sample-detector distance $\left(\mathrm{R}_{2}\right)$ is 
$4.39 \mathrm{~m}$, which gives a magnification of $\left.\mathrm{M}=\left(\mathrm{R}_{1}+\mathrm{R}_{2}\right) / \mathrm{R}_{1}\right)=6.3$. The resolution of the image is set by the imaging plate scanner to $50 \mu \mathrm{m}$. This allows resolution of details down to $\sim 8 \mu \mathrm{m}$. The transverse coherence length is defined by $L=\lambda l / \sigma$, where $\lambda$ is the wavelength of the source, $l$ the source to observation distance and $\sigma$ the source size. For a $1 \mu \mathrm{m}$ source size, $15 \mathrm{keV}$ X-rays and $5.2 \mathrm{~m}$ distance then $L \sim 400 \mu \mathrm{m}$.

Details of the body of the sample are clearly observed (see Figure 3). Edge enhancement is clearly observed in different parts of the sample, such as the legs (100-300 $\mu \mathrm{m}$ size).

The image has a relatively high noise level due to the imperfect experimental conditions. The aim is to experimentally show that phase-contrast imaging experiments can be done using the LWFA at the ALPHA-X facility and that the expected XPCi features are clearly observed in the image. Development of real world applications will be discussed in the next section.

Another interesting feature of the betatron X-ray beam is its femtosecond pulse duration. This ultrashort duration can be used in a wide variety of applications where motion blur is a problem. This is not possible with conventional synchrotron sources.

Further development of this tabletop synchrotron-like source and the scalability to $\mathrm{kHz}$ systems will enable the use of the LWFA in combination with more advanced phase-contrast imaging schemes ${ }^{3}$ and make them more commonly and commercially available. This will allow tomography of complex objects in a few seconds and perform also real-time XPC of objects. This shows the potential of the betatron X-ray beam for biomedical applications.

\section{MONTE CARLO SIMULATIONS FOR PHASE-CONTRAST IMAGING OF THIN BONES}

The scalability in energy of the betatron X-ray beams allows for phase-contrast imaging of more dense biological materials. For instance phase-contrast imaging of bone requires a critical energy of $\sim 50 \mathrm{keV}$ which can be achieved with the LWFA. It has been shown that the interaction of the electrons with the laser pulse ${ }^{21}$ in the bubble region can lead to a resonant enhancement of the betatron amplitude and therefore a higher critical energy of the betatron X-ray beams $(50-450 \mathrm{keV})$. This energy can be achieved in $100 \mathrm{TW}$-scale laser systems like the ASTRA-GEMINI laser at the Rutherford Appleton Laboratory.

In particular, phase-contrast imaging of micron size cracks in bone using the LWFA would be an interesting biomedical application. These micro-cracks are related to osteoporosis, bone stress, which can increase fragility. Detecting and monitoring their growth can help to predict and prevent larger fractures.

To study the feasibility and the resolution limit of the betatron X-ray beams from a LWFA for bone micro-crack phasecontrast imaging, we have performed simulations using the Monte Carlo radiation transport FLUKA ${ }^{22,23}$ code. For this, a refraction model based on Snell's law has been developed and included in the code ${ }^{24}$.

The parameters of the source used in the simulations are similar to the ones measured on the ALPHA-X beam line. As indicated above, for phase-contrast imaging of a bone $\sim 50 \mathrm{keV}$ would be required. However, as a first step and proof of principle, a thin section of $200 \mu \mathrm{m}$ of bone can be used.

The energy spectrum used in the simulations is a synchrotron-like spectrum similar to that in a conventional synchrotron:

$$
E_{c} \propto \int_{E / E_{c}}^{\infty} K_{5 / 3}(\xi) d \xi,
$$

where $K_{5 / 3}$ is the modified Bessel function of the second kind. The critical energy is set to $15 \mathrm{keV}$ (see Figure 4 ) and the number of particles used for each simulation was $10^{9}$. To reduce the computational time, the divergence was set to $2 \mathrm{mrad}$ which corresponds to the detector active area in the simulations. The source-to-sample distance is set to $30 \mathrm{~cm}$ and the sample-to-detector distance to $80 \mathrm{~cm}$. The bone model used in the simulation is a rectangular $200 \mu \mathrm{m}$ thick bone tissue. A single air micro-crack is placed in the bone center.

Simulated phase-contrast images for different thickness are shown in figure 5 . The thinnest micro-crack observed with FLUKA corresponds to a radius of $1 \mu \mathrm{m}$.

The corresponding convoluted images for $13 \mu \mathrm{m}$ pixel size (typical CCD pixel size) are shown in figure 6 . 


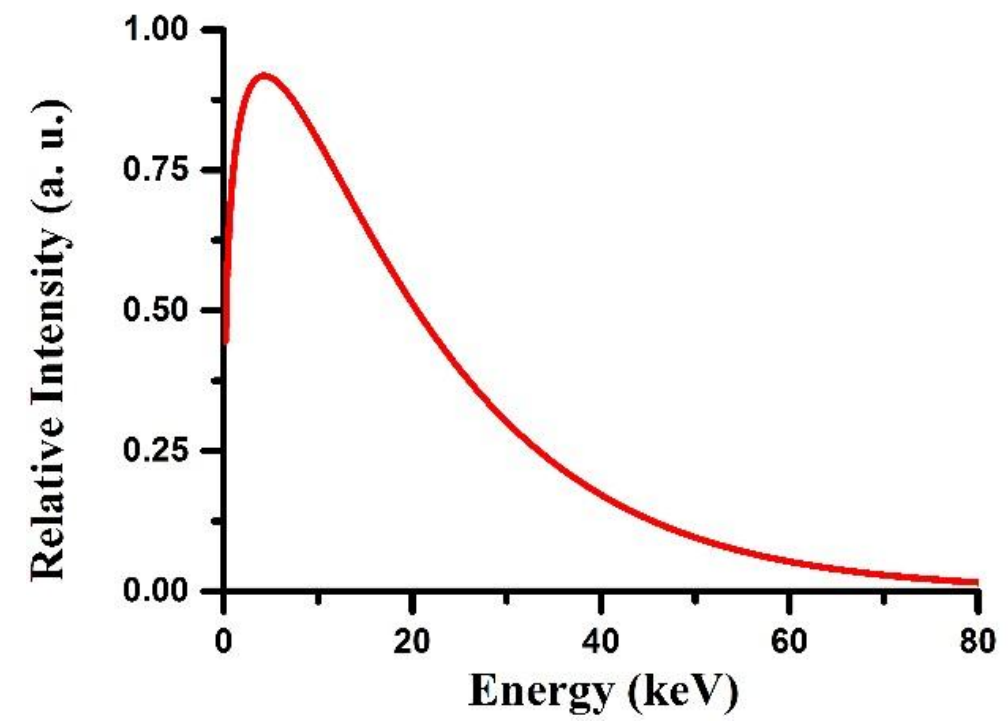

Figure 4. Synchrotron-like spectrum used for the phase-contrast FLUKA simulations. The critical energy is $15 \mathrm{keV}$ corresponding to a peak energy of about $4 \mathrm{keV}$.
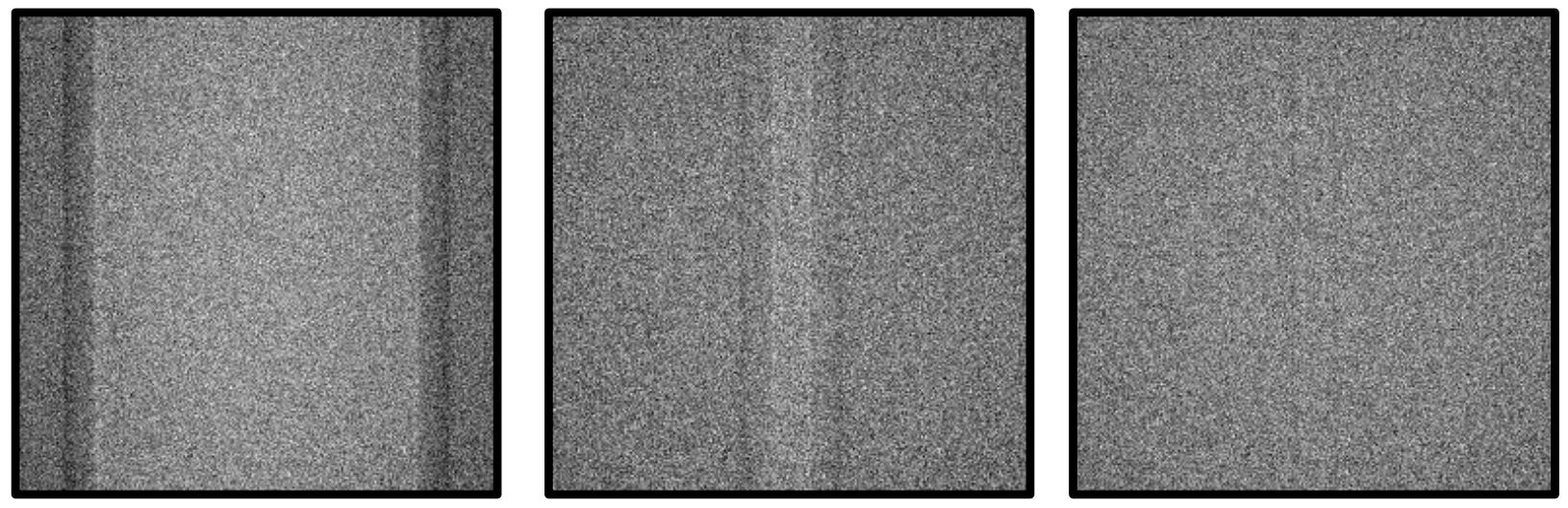

Figure 5. Simulated phase-contrast images for bone micro-cracks of 25,5 and $1 \mu$ m radius size respectively from left to right.
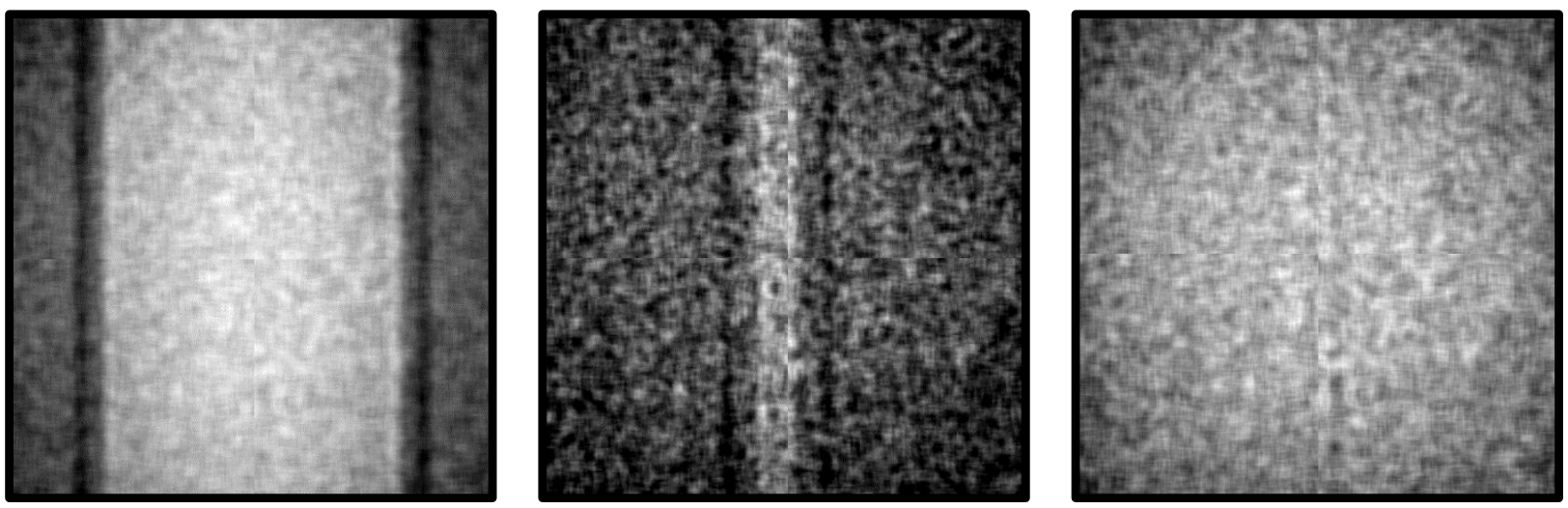

Figure 6. Corresponding convoluted images for $13 \mu \mathrm{m}$ pixel size. 

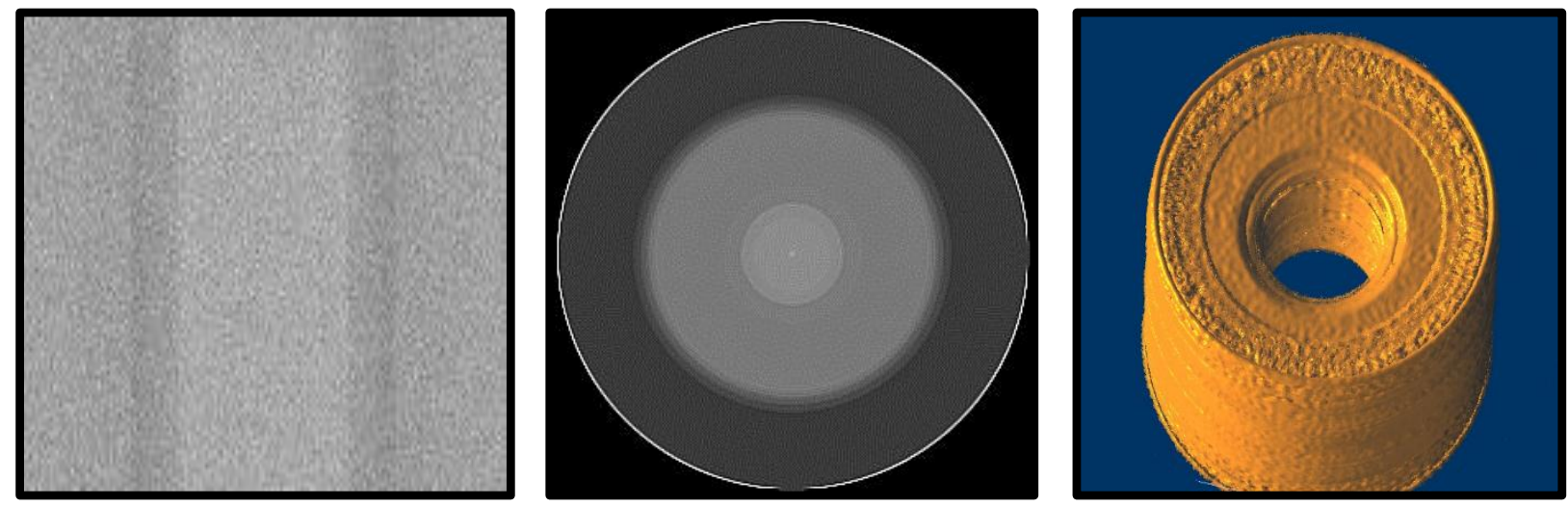

Figure 7. Examples of projection (left), tomogram (center) and 3D reconstruction (right) of a simulated $100 \mu \mathrm{m}$ diameter bone with a $40 \mu \mathrm{m}$ micro-crack.

With a sufficient number of projections it is possible to perform tomography and 3D reconstruction of micro-crack thin bones. To show this, 91 projections with an angle increment of 2 degrees have been simulated (see Figure 6). The bone diameter is $100 \mu \mathrm{m}$ and the micro-crack diameter is $40 \mu \mathrm{m}$. For processing the projections to obtain the tomograms, an inhouse developed tomography software based on filtered back projection algorithm is used while the 3D reconstruction used the image $\mathrm{J}^{25} 3 \mathrm{D}$ volume viewer plugin.

\section{CONCLUSIONS}

We have characterized the betatron X-ray beams at the ALPHA-X laser wakefield accelerator facility at the University of Strathclyde and performed phase-contrast imaging of a biological specimen using this source. The results of this study demonstrate the suitability of the betatron X-ray beams for the realization of phase-contrast imaging experiments on the ALPHA-X beam line.

We have performed Monte Carlo FLUKA simulations to explore the feasibility and the limits of this source for a real biomedical application, that is, for the acquisition of phase-contrast images of micron size cracks in bone. We have also studied 3D reconstruction of a simple thin bone model.

The LWFA appears to be a suitable synchrotron source for microscopic phase-contrast imaging. Scalability to kHz systems will allow tomography of complex objects to be performed in few seconds.

\section{ACKNOWLEDGEMENTS}

We acknowledge support of the U.K. EPSRC (grant no. EP/J018171/1), the EC's LASERLAB-EUROPE (grant agreement no. 284464, Seventh Framework Programme), EuCARD-2 (grant no. 312453, FP7) and the Extreme Light Infrastructure (ELI) European Project. We thank D. Clark and T. McCanny for technical support.

\section{REFERENCES}

[1] Roentgen, W.C., "On a new kind of rays," Nature, 1896. 53: p. 274-276.

[2] Munro, P.R.T., et al., "Design of a novel phase contrast X-ray imaging system for mammography," Physics in medicine and biology, 2010. 55(14): p. 4169-4185.

[3] Olivo, A. and R. Speller, "A coded-aperture technique allowing x-ray phase contrast imaging with conventional sources," Applied Physics Letters, 2007. 91(7).

[4] Mangles, S.P.D., et al., "Monoenergetic beams of relativistic electrons from intense laser-plasma interactions," Nature, 2004. 431(7008): p. 535-538.

[5] Geddes, C.G.R., et al., "High-quality electron beams from a laser wakefield accelerator using plasma-channel guiding," Nature, 2004. 431(7008): p. 538-541. 
[6] Faure, J., et al., "A laser-plasma accelerator producing monoenergetic electron beams," Nature, 2004. 431(7008): p. 541-544.

[7] Kneip, S., et al., "Bright spatially coherent synchrotron X-rays from a table-top source," Nat Phys, 2010. 6(12): p. 980-983.

[8] Tajima, T. and J.M. Dawson, "Laser Electron Accelerator," Physical Review Letters, 1979. 43(4): p. $267-270$.

[9] Strickland, D. and G. Mourou, "Compression of amplified chirped optical pulses," Optics Communications, 1985. 55(6): p. 447-449.

[10] Wiggins, S.M., et al., "High quality electron beams from a laser wakefield accelerator," Plasma Physics and Controlled Fusion, 2010. 52(12): p. 124032.

[11] Brunetti, E., et al., "Low Emittance, High Brilliance Relativistic Electron Beams from a Laser-Plasma Accelerator," Physical Review Letters, 2010. 105(21): p. 215007.

[12] Jaroszynski, D.A., et al., "Radiation sources based on laser-plasma interactions," Phil. Trans. R. Soc. A 364, 689710 (2006).

[13] Pukhov, A. and J. Meyer-ter-Vehn, "Laser wake field acceleration: the highly non-linear broken-wave regime," Applied Physics B, 2002. 74(4-5): p. 355-361.

[14] Rousse, A., et al., "Production of a keV X-Ray Beam from Synchrotron Radiation in Relativistic Laser-Plasma Interaction," Physical Review Letters, 2004. 93(13): p. 135005.

[15] Jackson, J.D., "Classical Electrodynamics" (John Wiley, 1999).

[16] Jaroszynski, D.A., et al., "The Strathclyde terahertz to optical pulse source (TOPS)," Nuclear Instruments and Methods in Physics Research Section A: Accelerators, Spectrometers, Detectors and Associated Equipment, 2000. 445(1-3): p. 317-319.

[17] Shanks, R. P., 2012: "Comprehensive Characterisation of Laser Plasma Wakefield Accelerated Electrons," Department of Physics. University of Strathclyde.

[18]Llopart, X., et al., "Timepix, a 65k programmable pixel readout chip for arrival time, energy and/or photon counting measurements," Nuclear Instruments and Methods in Physics Research Section A: Accelerators, Spectrometers, Detectors and Associated Equipment, 2007. 581(1-2): p. 485-494.

[19] Wilkins, S.W., et al., "Phase-contrast imaging using polychromatic hard X-rays," Nature, 1996. 384(6607): p. 335-338.

[20] Kneip, S., et al.," X-ray phase contrast imaging of biological specimens with femtosecond pulses of betatron radiation from a compact laser plasma wakefield accelerator," Applied Physics Letters, 2011. 99(9).

[21] Cipiccia, S., et al., "Gamma-rays from harmonically resonant betatron oscillations in a plasma wake," Nat Phys, 2011. 7(11): p. 867-871.

[22] A. Ferrari, P.R.S., A. Fasso`, and J. Ranft, "FLUKA: A multi-particle transport code," CERN-2005-10 (2005), INFN/TC_05/11, SLAC-R-773.

[23] Böhlen, T.T., et al., "The FLUKA Code: Developments and Challenges for High Energy and Medical Applications," Nuclear Data Sheets, 2014. 120(0): p. 211-214.

[24] Cipiccia, S., et al., "Inclusion of coherence in Monte Carlo models for simulation of x-ray phase contrast imaging," Optics Express, 2014. 22(19): p. 23480-23488.

[25] Schneider, C.A., et al., "NIH Image to ImageJ: 25 years of image analysis," Nature Methods 9, 671-675, 2012. 